\title{
Quiste óseo aneurismático del seno esfenoidal. A propósito de un caso
}

\author{
M. Rivero-Garvía; C.J. Saldaña; G.R. Boto; A. Pérez-Zamarrón; M.T. De Miguel; S. Gil-Robles y P. Mata
}

Servicio de Neurocirugía. Hospital Universitario Clínico San Carlos. Madrid.

\section{Resumen}

El quiste óseo aneurismático es una patología infrecuente que asienta generalmente en las metáfisis de huesos largos y vértebras. La afectación del seno esfenoidal es excepcional. Su origen se encuentra aún por definir, pero podemos considerarlos como un fenómeno vascular secundario a una lesión del hueso subyacente. Puede asociarse a otras enfermedades, presentes de forma metacrónica o sincrónica en distintas localizaciones de un mismo sujeto, como la displasia fibrosa poliostótica o los tumores de células gigantes. En este artículo se expone el caso de un paciente que presenta un quiste óseo aneurismático en el seno esfenoidal y alteración de la visión.

PALABRAS CLAVE: Quiste óseo aneurismático. Seno esfenoidal. Lesión ósea benigna.

\section{Summary}

Aneurysmal bone cyst is an uncommon lesion that occurs mainly in long bones and vertebrae, being the location in the sphenoid sinus extremely rare. Its origin is unknown, but it can be considered as a vascular phenomenon secondary to a primary lesion. Other primary diseases that may be associated to aneurysmal bone cyst are polyostotic fibrous dysplasia and giant-cell tumors. We report the case of a patient with an aneurysmal bone cyst in the sphenoid sinus causing vision dysfunction.

KEY WORDS: Aneurysmal bone cyst. Sphenoid sinus. Benign bone lesion.

\section{Introducción}

Los quistes óseos aneurismáticos son lesiones benignas, no invasivas, pero de rápido crecimiento. Su frecuencia se cifra alrededor del $1-2 \%$ de los tumores óseos primarios,

Recibido: 26-10-04. Aceptado: 13-11-04 localizados preferentemente en las metáfisis de huesos largos y los arcos posteriores vertebrales. La afectación craneal del quiste óseo aneurismático ocurre en el 3\% de los $\operatorname{casos}^{4,7,8}$. Predominan en el sexo femenino, entre la segunda y tercera década de la vida. Su etiopatogenia se encuentra en estudio, pero se han implicado varias alteraciones cromosómicas en su génesis como las traslocaciones: $\mathrm{t}(16 ; 17)$ (q22;p13) ó $\mathrm{t}(11 ; 16)$ (q13;q22-q23) ${ }^{5}$. Algunos autores sostienen que el quiste óseo aneurismático es siempre secundario a un tumor óseo que se destruye totalmente al formarse el lecho vascular aneurismático. La clasificación más utilizada, sin embargo, no tiene en cuenta esta circunstancia y los divide según la coexistencia o no de otras enfermedades óseas asociadas como: displasia fibrosa, tumor de células gigantes o condrosarcoma, entre otras. El síntoma más frecuente de presentación es el dolor, y la característica radiológica más notable es el edema perilesional reactivo a su rápido crecimiento. Se pueden elegir varias líneas de tratamiento (curetaje, resección en bloque, radioterapia...) sin que exista una posición unánime en cuanto al considerado de elección.

\section{Caso clínico}

El caso que presentamos corresponde a una mujer de 30 años. sin antecedentes personales relevantes, que consultó por un cuadro inicial de cefalea hemicraneal derecha pulsátil de dos meses de duración que progresó hasta convertirse en holocraneal. Dicha cefalea presentaba características de organicidad y era resistente al tratamiento convencional (antiinflamatorios no esteroideos y triptanos). Además, se acompañaba de visión borrosa, más acusada en ojo izquierdo, dos episodios de diplopia autolimitada y episodios ocasionales de ptosis palpebral izquierda. En la exploración neurológica destacaba un defecto pupilar aferente en el ojo izquierdo con disminución de la agudeza visual de $0.1 \mathrm{y}$ de 0.25 en el ojo derecho. El resto de los pares craneales no presentaban alteraciones a la exploración. El estudio hormonal no reveló ninguna alteración funcional. En la tomografía computarizada (TC) craneal realizada se encontró una lesión quística, no calcificada, que erosionaba 
Figura 1. TC craneal. Se aprecia una lesión isodensa, quística que erosiona el seno esferoidal y las paredes laterales de la silla turca. el seno esfenoidal y las paredes laterales de la silla turca (fig. 1). La resonancia magnética (RM) cerebral confirmó la existencia de una tumoración de 4 × 3 × $3 \mathrm{~cm}$. en la región del seno esferoidal, que destruía, erosionaba y expandía las paredes del seno y el propio clivus. La lesión producía compresión y desplazamiento craneal de la glándula hipofisaria y del quiasma. Se mostraba isointensa en las secuencias potenciadas en $T_{1}$ e hiperintensa en secuencias $T_{2}$, con niveles líquido-líquido en su interior, aspecto tabicado $\mathrm{y}$ realce periférico en las secuencias post-contraste. Además, existía esclerosis del hueso esfenoidal circundante (fig. 2).

Bajo anestesia general se realizó un abordaje sublabial transesfenoidal, resecándose parcialmente la lesión en su parte central, con intención de descomprimir el quiasma óptico.
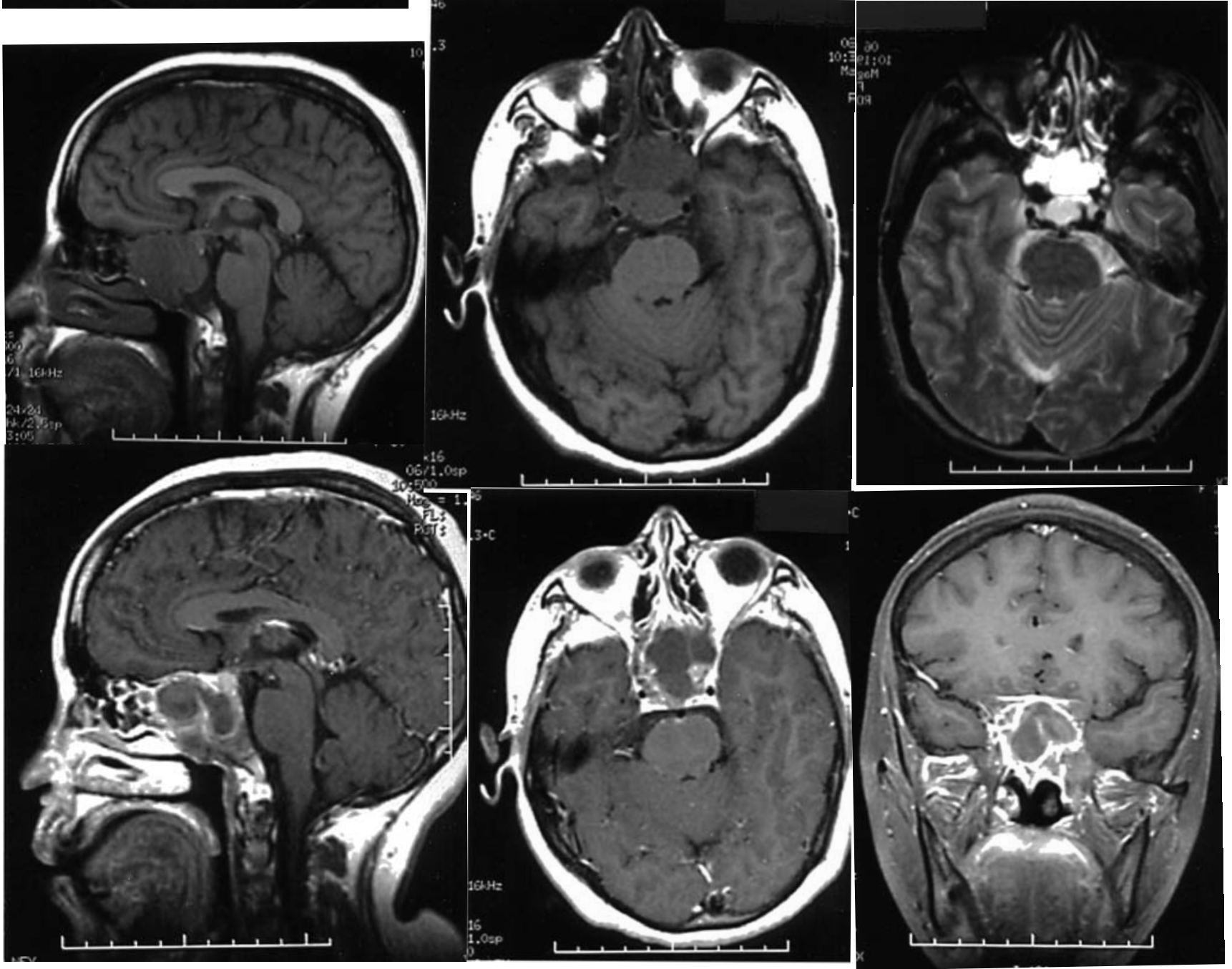

Figura 2. Superior: RM cerebral sin contraste donde se observa una lesión de alrededor de 4 cm de diámetro máximo anteroposterior, situada en la región del seno esfenoidal. isointensa en $T_{1}$ (izquierda y centro) e hiperintensa en $T_{2}$. La lesión produce compresión y desplazamiento craneal del quiasma y de la glándula hipofisaria. Inferior: RM cerebral post-contraste que el aspecto tabicado y multiquístico de la lesión. con niveles líquido-líquido en su interior y realce periférico con el contraste. 

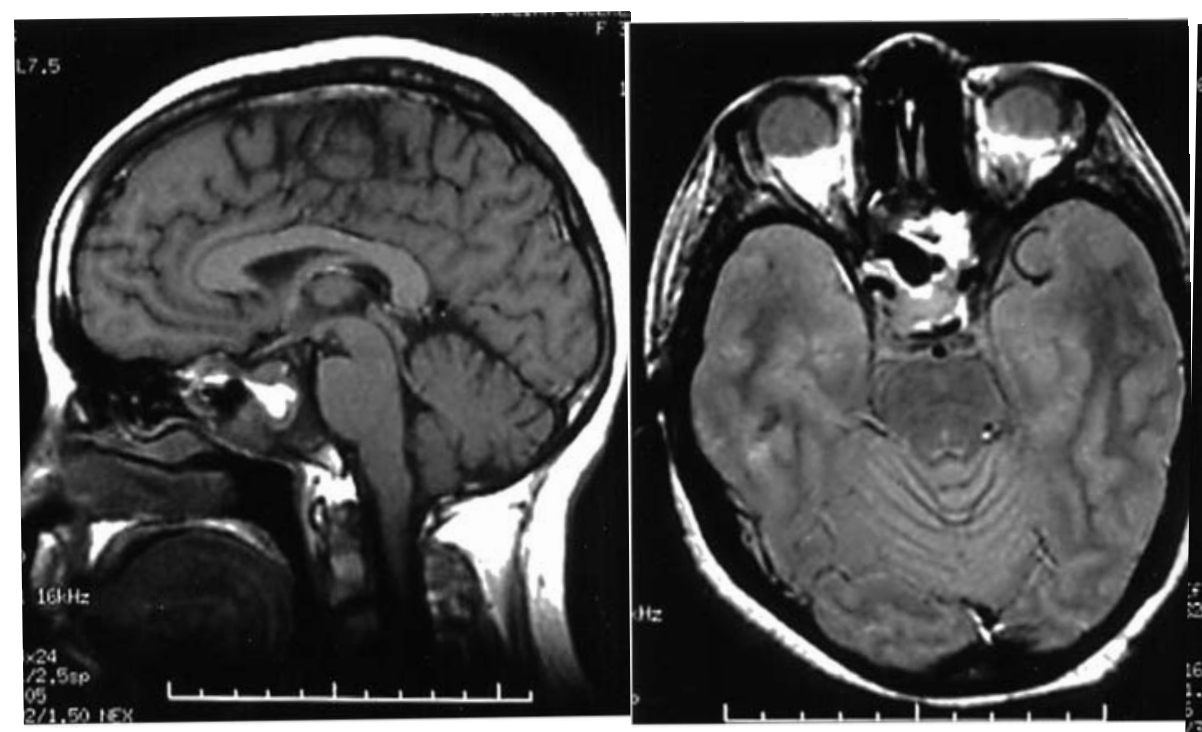

2005; 16: 365-368

Figura 3. RM cerebral postquirúrgica que pone de manifiesto la resección parcial con adecuada descompresión de la vía óptica.

En el análisis anatomopatológico se confirmó la presencia de un tejido de aspecto benigno parcialmente mineralizado, con presencia de osteoblastos y de trabéculas irregulares formando grandes lagos venosos, compatible con quiste óseo aneurismático.

En la RM de control posquirúrgica, se aprecia una adecuada descompresión del quiasma óptico con lesión residual en ambas regiones laterales y celdas etmoidales (fig. 3).

La exploración neuroftalmológica realizada a los tres meses confirma que la agudeza visual de la paciente era prácticamente normal (O.D.: 1 y O.I.: 0.9).

\section{Discusión}

El quiste óseo aneurismático representa del 1\% al 2\% de los tumores óseos primarios ${ }^{4,7,8}$. Como ya mencionamos en la introducción, bajo el punto de vista epidemiológico, afecta a mujeres menores de 30 años y la localización craneal se reduce al 3\%, siendo extremadamente rara su ubicación en el seno esfenoidal. Se clasifican como primarios o secundarios dependiendo de la coexistencia de patología ósea asociada. en cualquier lugar del organismo, tales como: displasia fibrosa, tumores de células gigantes condrosarcomas, etc ${ }^{4,6,7}$. El caso que nos atañe se puede considerar una lesión primaria, pues no ha podido demostrarse la asociación con otras enfermedades óseas en esta paciente, al menos hasta el momento.

$\mathrm{Su}$ crecimiento rápido, pero en lugares de difícil exploración. repercute en su presentación clínica solapada. Los de localización paraselar, como el nuestro, pueden presentar cefaleas, congestión nasal, parálisis de nervios craneales o pérdida de agudeza visual. Los síntomas endocrinos y la disfunción hormonal suelen ser excepcionales ${ }^{7}$, pues la lesión no invade el tejido limítrofe sano, respetando la hipófisis, como en nuestro caso. A pesar de su histología benigna, su agresividad local conduce en ocasiones a la recidiva.

Para la diferenciación de otras lesiones que asientan en el seno esfenoidal o región selar (mucoceles, osteomas, displasias fibrosas, tumores hipofisarios, condrosarcomas, adenocarcinomas...), recurrimos a las pruebas de imagen y al diagnóstico histopatológico ${ }^{5}$. Los quistes óseos aneurismáticos se presentan típicamente en las radiografías simples de cráneo como una lesión única, ocasionalmente lobulada, (adoptando, en ese caso, un patrón de "pompas de jabón", característico, pero no patognomónico), de carácter lítico y expansivo, con ribete óseo periférico escleroso ${ }^{1,2,7}$. En la TC craneal aparecen como lesiones hipo o hiperdensas (dependiendo de la concentración proteica del líquido quístico), generalmente multiloculadas, que pueden tener realce periférico con la administración de contraste ${ }^{1,2,6}$. La RM cerebral característica es aquélla en la que encontramos lesiones quísticas de intensidad heterogénea, con múltiples niveles líquido-líquido debido a la sangre no coagulada de su interior; la imagen en "panel de abeja" en secuencia $T_{1}$ tras la administración de gadolinio es producida por los múltiples tabiques internos, equivalente a la de "pompas de jabón" en secuencias $\mathrm{T}_{2}^{1,4}$. En la angiografía se aprecian múltiples lechos vasculares anómalos ${ }^{1}$.

Debido a la localización esferoidal, el diagnóstico diferencial debería realizarse con el mucocele. pues tanto su presentación clínica como su rango de edad etario son muy similares. Sin embargo, en el mucocele existen antece- 
dentes de infecciones de repetición, traumatismos, cirugía o pólipos nasales, que nos lleven a sospechar una obstrucción del drenaje parasinusal. En las pruebas de imagen (TC y RM), el mucocele también puede presentar un realce periférico tras la administración de contraste en la fase inflamatoria aguda ${ }^{4,6,7}$. Sin embargo, la presencia de una lesión tabicada con niveles líquido-líquido y la esclerosis del hueso subyacente en los estudios radiológicos sugieren el diagnóstico de quiste óseo aneurismático.

Entre las opciones terapéuticas se encuentra la resección quirúrgica (simple evacuación, resección en bloque), el curetaje, la crioterapia, la radioterapia. la embolización arterial selectiva coadyuvante o como tratamiento, y la escleroterapia con corticoides. La mayoría de los autores abogan por la resección quirúrgica, reservando la radioterapia para las recidivas, puesto que existen múltiples antecedentes en la literatura de sarcomas secundarios a radiación sobre estas lesiones. Sin embargo, Feigenberg et $\mathrm{al}^{3}$, han publicado una serie de casos tratados con bajas dosis de radioterapia como primera opción tras confirmación histológica del diagnóstico, sin encontrar mayor incidencia de tumores secundarios. Boriani et al. ${ }^{2}$ proponen la embolización selectiva para quistes óseos aneurismáticos localizados en segmentos móviles de la columna vertebral, donde el riesgo de sangrado ha de tenerse en cuenta.

En nuestro caso, el tratamiento elegido fue la resección quirúrgica más amplia posible, con el objetivo de conseguir un diagnóstico histológico y descomprimir la vía óptica.

\section{Bibliografía}

1. Asaumi. J., Konouchi, H., Hisatomi, M., et al.: MR features of aneurismal bone cyst of the mandibule and characteristics distinguishing it from other lesions. Eur J Radiol 2003;
45: 108-112.

2. Boriani, S., De Iure, F., Campanacci, L., et al: Aneurysmal bone cyst of the mobile spine: report on 41 cases. Spine 2001; 26: 27-35.

3. Feigenberg, S.J., Marcus, R.B. Jr., Zlotecki, R.A., Scarborough, M. T., Berrey, B.H., Enneking, W.F.: Megavoltage radiotherapy for aneurismal bone cysts. Int J Radiat Oncol Biol Phys. 2001 : 49: 1243-1247.

4. Gan, Y.C., Mathew, B., Salvage, D., Crooks, D.: Aneurysmal borre cyst of the sphenoid sinus. Br J Neurosur 2001; 15: 51-54.

5. Herens, C., Thiry, A., Dresse, M.F., et al: Translocation $(16 ; 17)(q 22 ; p 13)$ is a recurrent anomaly of aneurysmal bone cysts. Cancer Genet Cytogenet 2001; 127: 83-84.

6. Idbal, J., Kanaan, I., Al Homsi, M.: Non-neoplastic cystic lesions of the sellar region presentation, diagnosis and management of eight cases and reviev of the literature. Acta Neurochir (Wien) 1999; 141: 389-398.

7. Itshayek, E., Spector, S., Gomori, M., Segal, R.: Fibrous dysplasia in combination with aneurysmal bone cyst of the occipital bone and the clivus: case report and review of the literature. Neurosurgery 2002; 51: 815-818.

8. Saito, K., Fukuta, K., Takahashi, M., Seki, Y., Yoshida, J.: Bening fibroosseous lesions involving the skull base, paranasal sinuses, and nasal cavity. Report of two cases. J Neurosurg 1998; 88: 1116-1119.

Rivero-Garvía, M.; Saldaña, C.J.; Boto, G.R.; Pérez Zamarrón, A.; De Miguel, M.I.; Gil-Robles, S.; Mata, P.: Quiste óseo aneurismático del seno esfenoidal. A propósito de un caso. Neurocirugía 2005; 16: 365-368.

Correspondencia postal: Mónica Rivero Garvía. C/ Profesor Martín Lagos s/n. 28040 Madrid. 